The Egyptian Journal of Hospital Medicine (July 2019) Vol. 76 (1), Page 3224-3231

\title{
Right Ventricular Outflow Tract Time Velocity Integral: A Noninvasive Echocardiographic Parameter of Right Ventricular Systolic Function In Cardiomyopathic Patients
}

\author{
Asmaa A. Ali
}

Cardiology Department, El Zahraa University Hospital, Faculty of Medicine for Girls, Al-Azhar University

Email: dr.aahmedali@gmail.com

\begin{abstract}
Background: Right ventricular dysfunction (RVD) is considered a powerful predictor in patients with left ventricular dysfunction. So, we should evaluate right ventricular (RV) function in patients with cardiomyopathy to predict RVD and avoid progression of the disease. Due to the burden of disease, an easily utilized screening tool for identifying such patients would be helpful to facilitate assessment for advanced therapies.

Aim of the work: to assess RVOT-TVI and its correlation with RV systolic function by different echocardiographic modalities in patients with dilated cardiomyopathy.

Methods and study population: 70 cases (40 myopathic patients and 30 age and sex-matched healthy persons as a control group). All cases underwent complete TTE study to measure LV and RV dimensions, volumes and functions. In addition, TAPSE and RVFAC. RVOT-VTI is calculated. The LV and RV longitudinal strain was also assessed using 2D STE analysis.

Results: This study included 70 individuals divided into two groups; G "I" represented 40 patients with cardiomyopathy and G "II" represented 30 age-and sex-matched healthy subjects as control. Patients with DCM had significantly increased RV volumes $(p<0.001)$ and significantly lower RV systolic function. In addition, there was significant decreased RVOTTVI in cardiomyopathic patients group versus normal group. Moreover, The RVOT-VTI had a significant correlation with different indices of RV systolic function (RVEF $(\mathrm{r}=0.457, p<0.001)$, RV Sm $(\mathrm{r}=0.495, p<0.001)$ and 2D-RVGLS ( $\mathrm{r}=$ $0.453, p<0.001)$, \% FAC $(\mathrm{r}=0.398, p=0.001))$.

Conclusion: Right ventricular dysfunction is common in dilated cardiomyopathy. RVOT-VTI is a simple, non-invasive measure assess RV systolic function in cardiomyopathic patients.
\end{abstract}

Keywords: dilated cardiomyopathy, RV systolic function, RVOT time velocity integral.

\section{INTRODUCTION}

Congestive heart failure is always growing health care problem in the United States, as thousands deaths annually and millions yearly hospitalized ${ }^{[1,2]}$. Thus, accurate and timely prediction of high-risk profiles is important. As Idiopathic dilated cardiomyopathy (IDCM) is the second most common cause of heart failure (HF), which is not only caused by disease of the left ventricle (LV) but also the right ventricle (RV) and may play an important role in functional capacity of the patients than is often recorded ${ }^{[3]}$. Therefore, the identification of $\mathrm{RV}$ systolic dysfunction is of clinical importance however, its evaluation still challenging ${ }^{[4]}$. As its echocardiographic assessment is difficult because of its complex anatomy ${ }^{[5]}$. Right ventricular dysfunction (RVD) is considered a powerful predictor in patients with left ventricular dysfunction. So, we should evaluate right ventricular (RV) systolic function in patients with cardiomyopathy to predict early RVD and avoid progression of the disease [6].

Although the 2-D echocardiography is considered an important and integral tool for evaluation of patients with heart disease but most of its parameters focus on the left chamber function not the right chamber, namely the right ventricular (RV) systolic function. Early beliefs considered the right ventricle is less important than the left ventricle ${ }^{(7)}$. This is because the knowledge of RV function is not affected by the left ventricular (LV) function and less was known about the role of RV physiology and prognosis. Due to the burden of disease, an easily utilized screening tool for identifying such patients and to facilitate its timely assessment for advanced therapies is urgently needed ${ }^{[8]}$.

\section{AIM OF THE WORK}

Our study was designed to assess RVOT-TVI and its correlation with RV systolic function by different echocardiographic modalities in patients with dilated cardiomyopathy.

\section{METHODS}

\section{Study population}

We conducted a retrospective case-control study that enrolled 70 cases (40 myopathic patients and 30 age and sex-matched healthy persons as a control group). The study cases were selected from the Cardiology Outpatient Clinic at Al-Zahraa Hospital, Faculty of Medicine for Girls, Al-Azhar University, from March 2018 to April 2019. A person was considered to have DCM if dilated LV with systolic function less than $35 \%$. 
All subjects were informed about the purpose of the study and we obtained a verbal consent in presence of a third party and the study was approved by the Ethical Committee of Al-Azhar University, Cairo, Egypt.

Exclusion criteria included any one of the following: acute myocardial infarction, significant valvular disease affecting accurate estimation of forward cardiacoutput by RVOT-VTI (i.e. severe pulmonary regurgitation) [5], patients with rheumatic heart disease, congenital heart disease, other pre-existing cardiovascular disease like myocarditis due to infective etiology, significant arrhythmia, ischemic cardiomyopathy and poor echogenic window.

All the studied individuals were subjected to a full history with special emphasis on demographic data; age, sex and NYHA functional class assessment. Thorough clinical examination and systemic examination (chest, CVS, abdominal and CNS including blood pressure measurement) were performed.

\section{Standard echocardiography}

All cases underwent complete trans-thoracic echocardiography (TTE) study in both supine and left lateral position (including 2D, M mode, color flow and spectral Doppler as well as TDI, and speckle tracking) in the standard views (parasternal long axis, parasternal short axis, apical four, three and two chamber views) from all accessible windows were obtained with ECG physiosignal displayed with all detected echo-Doppler study with Loop recording of 2-3 cycles. All measurements were made over at least three cardiac cycles and the average value for each parameter was calculated using GE system XD CLEAR 9, Matrix probe M3S multi frequency $2.5 \mathrm{MHz}$, with the capability of tissue Doppler imaging and gray scale recording for speckle tracking study. All images were digitally stored for later off-line analysis at echoPac.GE version 210. All parameters were taken according to standards of the American Society of Echocardiography to measure LV dimensions and LV and RV functions.

Biplane LV end-diastolic and -systolic volumes were assessed from the apical 2- and 4-chamber images and LV ejection fraction was calculated using the biplane Simpson's formula. In addition, RV size, EF, tricuspid annular excursion (TAPSE) and RV end diastolic area (EDA), RV end systolic area (ESA) to measure RV fractional area change (RVFAC). RVOT-VTI (velocity time integral) is calculated by placing the pulsed Doppler sample volume in the outflow tract below the pulmonary valve and recording the velocity $(\mathrm{cm} / \mathrm{s})$. When the velocity signal is integrated with respect to time, the distance blood moves with each systole is calculated in $\mathrm{cm} /$ systole.

\section{Tissue Doppler Imaging (Tissue velocity echocardiography)}

Tissue Doppler cine loops were obtained from 3 beats in apical 4 chambers at depth of 14 with pulse repetition frequency at $1 \mathrm{kHz}$, Nyquest velocity range $\pm 16 \mathrm{~cm} / \mathrm{sec}$ and frame rate $99 \pm 9 \mathrm{~Hz}$. Standard apical 4-chamber images to obtain RV (S) wave of the lateral tricuspid annulus. The image sector width was set as narrow as possible to allow a frame rate acquisition greater than 90 frames/s to visualize one myocardial wall in order to obtain an optimal alignment between the wall and the ultrasound beam and to maximize frame rates. The gain settings, filters and pulse repetition frequency were adjusted to optimize color saturation and to avoid aliasing.

Two observers, blinded to the results of the echocardiographic RV function analysis, performed offline analyses. The LV and RV longitudinal strain was also assessed using 2D STE analysis with QRS onset as the reference point, applying a commercially available strain software package to the RV on echoPac version 210 . Images were acquired at 70-90 frames per second at endexpiration in the apical 4-chamber, 3-chamber and 2chamber views. Using the Automated Function Imaging software (AFI), a point-and-click approach was utilized to identify three anchor points (two basal and one apical), following which the software tracked the endocardial contour to assess RV function by speckle tracking. The LV 2D ST GS \% was obtained in all study cases automatically.

\section{Statistical analysis}

Statistical analysis was performed using the Statistical Package for Social Sciences (version 16.0; SPSS Inc., Chicago, IL, USA). Data were expressed as the mean \pm SD. Differences between groups were analyzed with unpaired $t$-test. Possible associations were assessed by Pearson and Spearman coefficients of correlation. $p$-value $<0.05$ was considered significant.

\section{RESULTS}

The enrolled population divided into patient group with mean age 56.3 years old and controlling group with mean age 41.9 years old. The most of the patient group were male. About $66 \%$ of the patient group were hypertensive, $28 \%$ were diabetic and $16 \%$ were hypercholestrolemic. 10 patients with NYHA functional class II and 30 patients with NYHA functional class III 
and most of them maintaining on medical treatment. Demographic and clinical characteristics of the enrolled population are summarized in table (1).

Table (1): Baseline demographic and clinical characteristics of the study population

\begin{tabular}{|c|c|c|}
\hline Variables & $\begin{array}{c}\text { Group I }(n= \\
\mathbf{4 0})\end{array}$ & $\begin{array}{c}\text { Group II } \\
(\mathbf{n}=\mathbf{3 0})\end{array}$ \\
\hline Age (years) & $56.3 \pm 8.3$ & $41.9 \pm 7.9$ \\
\hline Male sex (\%) & $31 / 40(70 \%)$ & $\begin{array}{c}25 / 30 \\
(75 \%)\end{array}$ \\
\hline Hypertension (\%) & $30 / 40(66.67 \%)$ & \\
\hline Diabetes Mellitus (\%) & $28 / 40(60 \%)$ & \\
\hline Hypercholesterolaemia(\%) & $16 / 40(20 \%)$ & \\
\hline Active Smoking (\%) & $26 / 40(53.3 \%)$ & $\begin{array}{l}15 / 30 \\
(50 \%)\end{array}$ \\
\hline $\begin{array}{l}\text { NYHA functional class } \\
\text { II/III }\end{array}$ & $10 / 30$ & \\
\hline $\begin{array}{l}\text { ACE-inhibitors or ARB } \\
\text { blocker }(\%)\end{array}$ & $40 / 40(100 \%)$ & \\
\hline Beta-blocker (\%) & $\begin{array}{c}35 / 40 \\
(83.33 \%) \\
\end{array}$ & \\
\hline Loop diuretic (\%) & $30 / 40(66.67 \%)$ & \\
\hline Spironolactone (\%) & $20 / 40(50 \%)$ & \\
\hline
\end{tabular}

As regard echocardiographic parameters of the LV, there was significantly increased LV dimensions and volumes but decreased $\mathrm{EF}$ whether by $2 \mathrm{D}$ or M-mode in cardiomyopathic patient group versus normal group by conventional echocardiography.

In addition, there was a significant lower LV GLS $\%$ in the patient group versus normal one (Table 2).
Table (2): Baseline echocardiographic parameters of the study population as regard LV parameters

\begin{tabular}{|c|c|c|c|c|}
\hline \multirow[t]{2}{*}{ Variable } & \multicolumn{2}{|c|}{ G I $(n=40)$} & \multicolumn{2}{|c|}{ G II $(n=30)$} \\
\hline & Mean & SD & Mean & SD \\
\hline IVSd (mm) & 8.95 & 2.23 & 8.45 & 1.45 \\
\hline LVIDd (mm) & 65.31 & 7.63 & 48.55 & 4.08 \\
\hline LVIDs (mm) & $\mathbf{5 3 . 9 0}$ & 7.55 & 30.00 & 4.22 \\
\hline $\begin{array}{c}\text { LVPWd } \\
(\mathbf{m m})\end{array}$ & 9.11 & 2.00 & 8.90 & 1.07 \\
\hline LVEF (\%) & 35.07 & 7.96 & 65.75 & 5.48 \\
\hline FS (\%) & 16.20 & 5.49 & 37.85 & 5.41 \\
\hline LV EDV & 160.01 & 47.20 & 70.62 & 14.92 \\
\hline LV ESV & 117.32 & 40.88 & 34.12 & 6.89 \\
\hline $\begin{array}{c}\text { 2D LVEF } \\
(\%)\end{array}$ & 30.17 & 18.10 & 56.58 & 13.83 \\
\hline LAD (mm) & 46.37 & 6.07 & 35.90 & 3.86 \\
\hline 2D-LVGLS (\%) & 7.23 & 3.20 & 19.48 & 2.69 \\
\hline
\end{tabular}

Abbreviations: IVSd, interventricular septal wall thickness in diastole; LVIDd, left ventricular internal dimension in diastole; LVIDs, left ventricular internal dimension in systole; LVPWd, left ventricular posterior wall thickness in diastole; LVEF, left ventricular ejection fraction; FS, fractional shortening; LAD, left atrial diameter; LVEDV , left ventricular end diastolic volume; LVESV, left ventricular end systolic volume; LV Sm, mitral annular systolic velocity by tissue Doppler; 2D-LVGLS, left ventricular global longitudinal strain measured by speckle tracking echocardiography.

The patients with DCM had significantly increased RV volumes but lower RV EF, \% FAC, TAPSE, RV Sm and 2D RVGLS as shown in figure (1) and table (3).

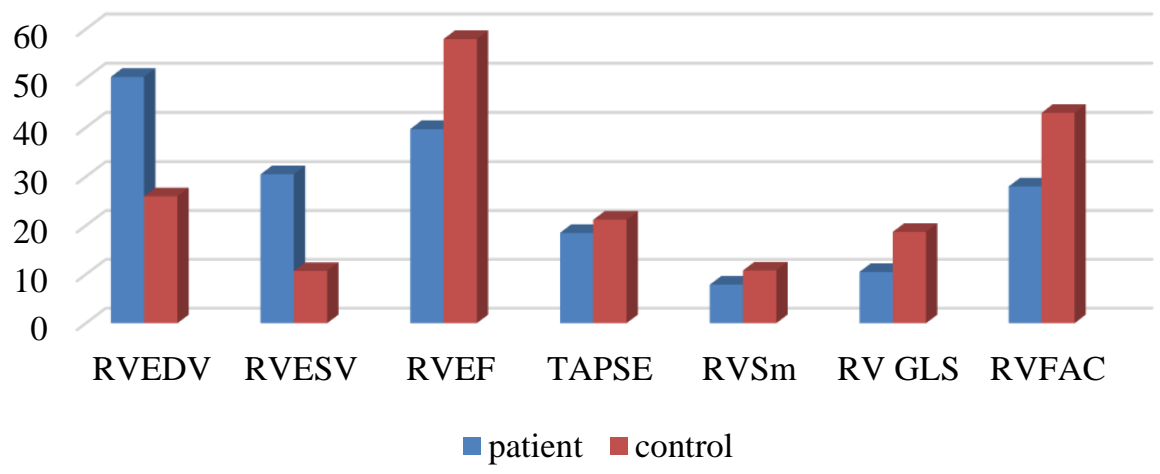

Figure (1): Comparison between group I and II as regard indices of RV systolic function by different echo modalities 
Table (3): Baseline echocardiographic parameters of the study population as regard RV parameters

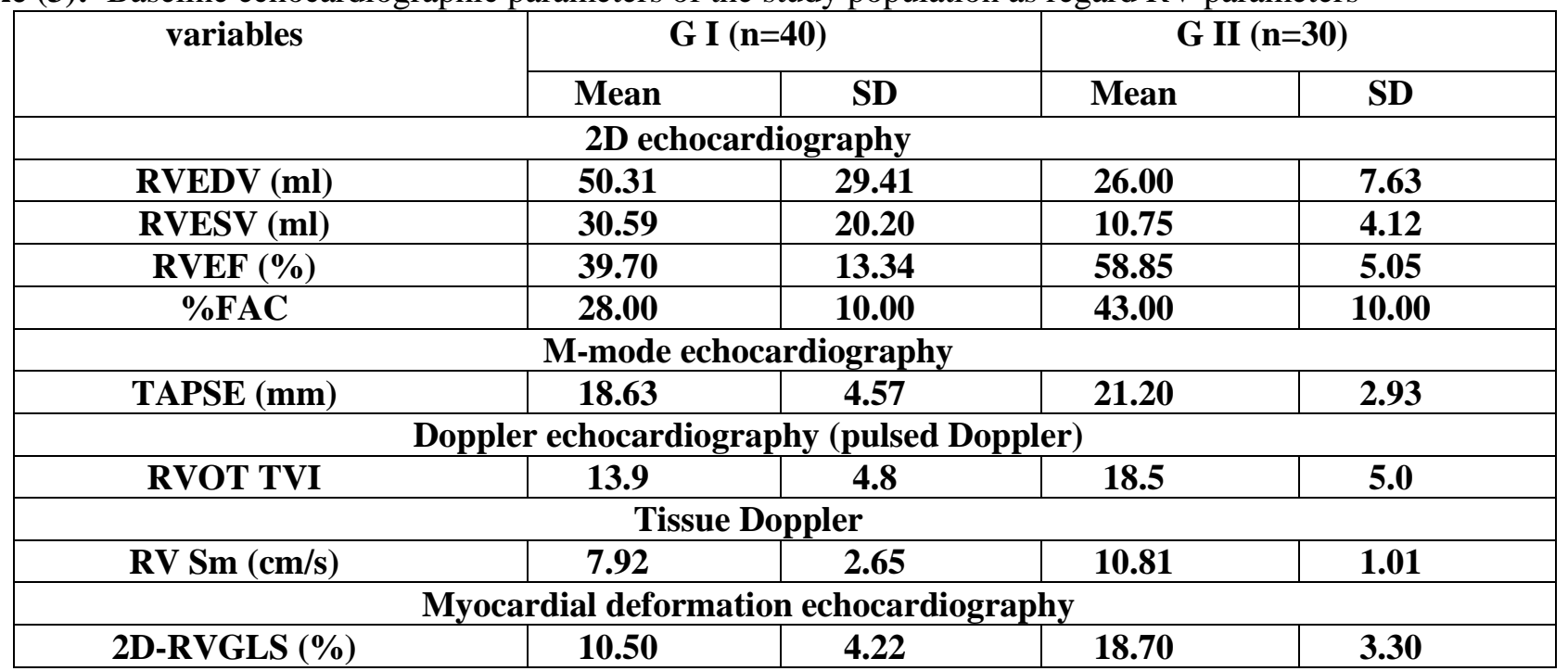

Abbreviations: RVEDV, right ventricular end diastolic volume; RVESV, right ventricular end systolic volume; RVEF, right ventricular ejection fraction; FAC, fractional area change; TAPSE, tricuspid annular plane systolic excursion; Sm, tricuspid annular systolic velocity measured by tissue Doppler; 2D-GLS, global longitudinal strain measured by 2D speckle tracking echocardiography; RVOT TVI, time velocity integral of right ventricular outflow tract.

Also, there was decreased RVOT-TVI in cardiomyopathic patient group versus normal group as shown in figure (2).
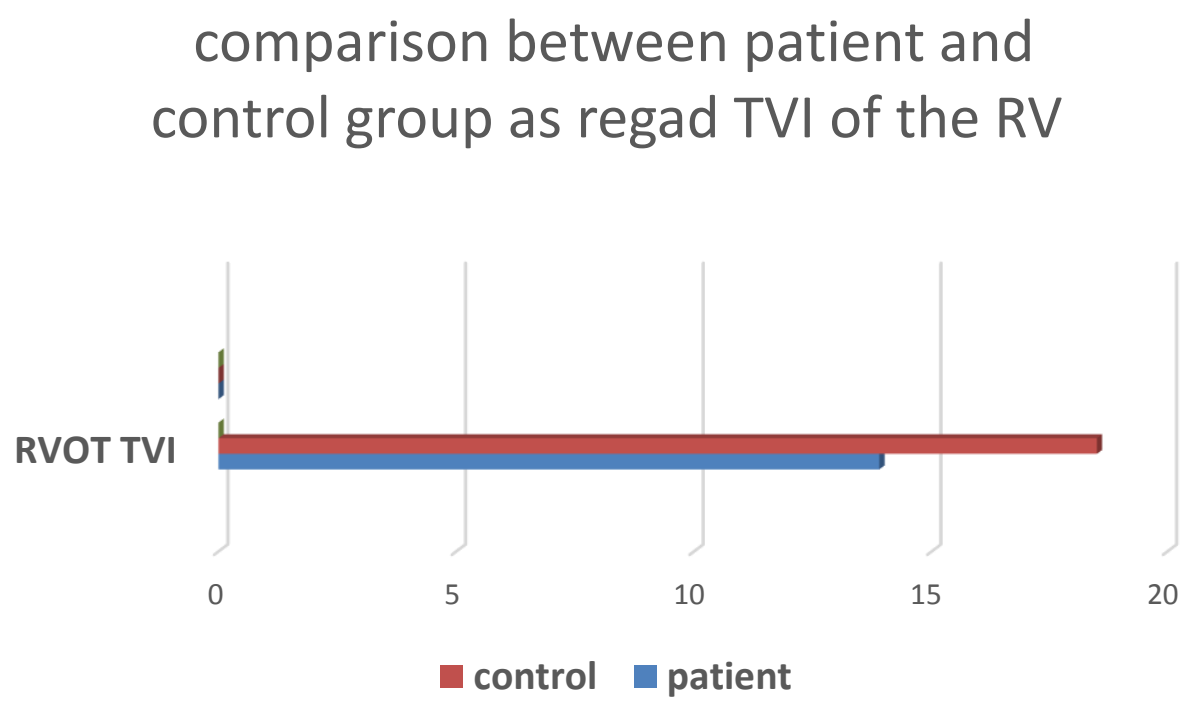

Figure (2): comparison between patient and control group as regard TVI of the RVOT

In comparison to control, there was significantly increased LV dimensions, volumes and EF whether by 2D or M-mode ( $\mathrm{p}$ value $=0.000$ ) in patients group versus normal group by conventional echocardiography. In addition, there was a significant lower TDI systolic velocity and LV GLS \% in the cardiomyopathic group versus normal one (Table 4). 
Table (4): Comparison between Baseline LV echocardiographic parameters in patient group versus normal controlling group.

\begin{tabular}{|c|c|c|c|c|c|}
\hline \multirow[t]{2}{*}{ Variable } & \multicolumn{2}{|c|}{ G I $(n=40)$} & \multicolumn{2}{|c|}{ G II $(n=30)$} & \multirow[t]{2}{*}{ P value } \\
\hline & Mean & SD & Mean & SD & \\
\hline IVSd (mm) & 8.95 & 2.23 & 8.45 & 1.45 & 0.29 \\
\hline LVIDd (mm) & 65.31 & 7.63 & 48.55 & 4.08 & 0.000 \\
\hline LVIDs (mm) & 53.90 & 7.55 & 30.00 & 4.22 & 0.000 \\
\hline LVPWd (mm) & 9.11 & 2.00 & 8.90 & 1.07 & 0.53 \\
\hline LVEF (\%) & 35.07 & 7.96 & 65.75 & 5.48 & 0.000 \\
\hline FS (\%) & 16.20 & 5.49 & 37.85 & 5.41 & 0.000 \\
\hline LV EDV & 160.01 & 47.20 & 70.62 & 14.92 & 0.000 \\
\hline LV ESV & 117.32 & 40.88 & 34.12 & 6.89 & 0.000 \\
\hline 2D LVEF (\%) & 30.17 & 18.10 & 56.58 & 13.83 & 0.000 \\
\hline LAD (mm) & 46.37 & 6.07 & 35.90 & 3.86 & 0.000 \\
\hline 2D-LVGLS (\%) & 7.23 & 3.20 & 19.48 & 2.69 & 0.000 \\
\hline
\end{tabular}

Patients with DCM had significantly increased RV volumes $(p<0.001)$ and significantly lower RV EF $(p<0.001), \%$ FAC $(p<$ $0.001)$, TAPSE $(p<0.01)$, RV Sm $(p<0.001)$, RVGLS 2D derived $(p<0.001)$ as shown in table (5). Also, there was significant decreased RVOT-TVI in cardiomyopathic patients group versus normal group.

Table (5): Comparison between Baseline LV echocardiographic parameters in patient group versus normal control group.

\begin{tabular}{|c|c|c|c|c|c|}
\hline \multirow[t]{2}{*}{ Variable } & \multicolumn{2}{|c|}{ G I $(n=40)$} & \multicolumn{2}{|c|}{ G II $(n=30)$} & \multirow[t]{2}{*}{ P value } \\
\hline & Mean & SD & Mean & SD & \\
\hline \multicolumn{6}{|c|}{ 2D echocardiography } \\
\hline RVEDV (ml) & $\mathbf{5 0 . 3 1}$ & 29.41 & 26.00 & 7.63 & $\mathbf{0 . 0 0 0}$ \\
\hline RVESV (ml) & 30.59 & 20.20 & 10.75 & 4.12 & 0.000 \\
\hline RVEF (\%) & 39.70 & 13.34 & 58.85 & 5.05 & 0.000 \\
\hline \%FAC & 28.00 & $\mathbf{1 0 . 0 0}$ & 43.00 & $\mathbf{1 0 . 0 0}$ & 0.000 \\
\hline \multicolumn{6}{|c|}{ M-mode echocardiography } \\
\hline TAPSE (mm) & 18.63 & 4.57 & 21.20 & 2.93 & 0.008 \\
\hline \multicolumn{6}{|c|}{ Doppler echocardiography (pulsed Doppler) } \\
\hline RVOT TVI & 13.9 & 4.8 & 18.5 & 5.0 & 0.0001 \\
\hline \multicolumn{6}{|c|}{ Tissue Doppler } \\
\hline RV Sm $(\mathrm{cm} / \mathrm{s})$ & 7.92 & 2.65 & 10.81 & 1.01 & 0.000 \\
\hline \multicolumn{6}{|c|}{ Myocardial deformation echocardiography } \\
\hline 2D-RVGLS (\%) & 10.50 & 4.22 & 18.70 & 3.30 & 0.000 \\
\hline
\end{tabular}

The RVOT time velocity integral had a significant correlation with different indices of RV systolic function (RVEF $(\mathrm{r}=0.457, p<0.001)$, RV Sm $(\mathrm{r}=0.495, p<0.001)$, and 2D-RVGLS $(\mathrm{r}=0.453, p<0.001)$, while with TAPSE $(\mathrm{r}=$ $0.397, \mathrm{p}=0.001)$ and $\%$ FAC $(\mathrm{r}=0.398, p=0.001))$.

Table (6): Correlation between RVOT-VTI and RV systolic function by different echo modalities

\begin{tabular}{|c|c|c|}
\hline Variables & $(\mathbf{r})$ & P value \\
\hline TAPSE & $\mathbf{0 . 3 9 7}$ & $\mathbf{0 . 0 0 1}$ \\
\hline FAC & $\mathbf{0 . 3 9 8}$ & $\mathbf{0 . 0 0 1}$ \\
\hline RVEF & $\mathbf{0 . 4 5 7}$ & $\mathbf{0 . 0 0 0}$ \\
\hline RV Sm & $\mathbf{0 . 4 9 5}$ & $\mathbf{0 . 0 0 0}$ \\
\hline RV GLS & $\mathbf{0 . 4 5 3}$ & $\mathbf{0 . 0 0 0 2}$ \\
\hline
\end{tabular}




\section{DISCUSSION}

Transthoracic echocardiography has been one of the most important and reliable used methods for the evaluation of patients with known cardiomyopathy. The important role of $\mathrm{RV}$ function in patients with reduced-EF HF has been known earlier since the initial studies on isotopic RVEF ${ }^{[9-10]}$. Examination of the right ventricle often remains neglected compared with that of the left ventricle, because of its complex anatomy and also, its retrosternal position. But, still many variables of RV systolic function and morphology have been shown to be predictors of adverse outcome in patients with HF ${ }^{[11-17]}$. In the presence of reduced$\mathrm{EF} \mathrm{HF}, \mathrm{RV}$ involvement is a distinctive feature of idiopathic DCM, independent of LV dysfunction $[18$, 19].

RV systolic function was assessed in our study by using tricuspid annular plane systolic excursion (TAPSE) with R V Dysfunction defined as TAPSE < $18 \mathrm{~mm}$ (as in Lindqvist's study), RV EF, RV FAC, RV TDI (S wave) and RV STE. In Lindqvist ${ }^{[20]}$ defined RV function only by TAPSE. However, TAPSE only measures tricuspid annular motion and may not accurately assess RV global or segmental contractile function and as many other parameters may be subjected to error secondary to translational cardiac motion. This limitation of course not present when using speckletracking-based strain imaging.

Therefore, this study demonstrated the use of quantifying RV systolic function also by 2-D strain (STE) measurement routinely in patients referred for echocardiographic evaluation for known dilated cardiomyopathy especially for patients with RV impairment. Measurement of myocardial strain using speckle-tracking echocardiography gives information about quantitative evaluation of ventricular systolic function ${ }^{[21]}$.

Strain imaging is sensitive to early impairments and even subclinical affection of systolic function that may develop despite the presence of normally appearing contractility by 2D imaging. Therefore, it is valuable for assessment of the RV. Longitudinal systolic strain measurement is also well suited to assess RV function because RV contractility predominantly occurs in the longitudinal plane ${ }^{[22]}$. Many previous studies have demonstrated the importance of measuring RV strain using speckle-tracking echocardiography in assessment of RV dysfunction ${ }^{[23-}$ 26].

In our study, we evaluated a novel parameter to assess RV systolic function using Doppler echocardiography of the RVOT from the parasternal short-axis view, which is RVOT-VTI. We found RVOTVTI to be a simple, quick and feasible parameter of RV systolic function. Because this area of the RV is less susceptible to ischaemia and regional RV abnormalities than other areas, such as the lateral free wall, which is often involved in RV infarction and can affect TAPSE even when global RV systolic function is preserved ${ }^{[27,28]}$. Furthermore, the RVOT-VTI, as a stroke volume surrogate, incorporates full RV ejection as opposed to TAPSE, which reflects RV basal motion only.

In our study, there was a surprise to find how well RVOT-VTI measurement differs between patients with RV systolic dysfunction as opposed to others with normal RV systolic function. RVOT-VTI also identified patients with reduced RV systolic function more accurately than tissue Doppler parameters such as tricuspid annular peaksystolic velocity $\left(\mathrm{S}^{\prime}\right)$.

In our study, RVOT-TVI well correlated with TAPSE (p value 0.001), RV FAC (p value 0.001) and RV EF ( $\mathrm{p}<0.001)$ by conventional echocardiography and with 2D strain (STE) as recent echocardiographic modalities ( $p$ value 0.0002 ).

Our studied patients were predominantly males (31out of 40), their mean age was 56 years old with systolic HF due to dilated cardiomyopathy (mean EF 35\%). Our findings demonstrated that extremely diminished RVOT-VTI was associated with RV systolic dysfunction as measured by other parameters. Moreover, RVO-TVTI provided prognostic information over ejection fraction, as it focuses on forward output, which might be normal even in patients with low ejection fraction ${ }^{[29]}$.

Our Study also agrees with previous one in 2012 on 41 patients with severe AS aged 80 years and proved that RVOT-VTI as a strong predictor of right ventricular dysfunction in elderly patients with severe aortic stenosis ${ }^{[30]}$.

Because RVOT-VTI is an easily obtainable measurement, so the RVOT-VTI may be a useful and accessible tool to identify RV systolic dysfunction in patients with dilated cardiomyopathy.

\section{CONCLUSION}

Right ventricular dysfunction is common in dilated cardiomyopathy. RVOT-VTI is a simple, non-invasive measure assesses RV systolic function in cardiomyopathic patients and is highly predictive of RV systolic dysfunction in these patients.

\section{RECOMMENDATIONS}

Further studies are needed to determine the value of RVOT-VTI compared to established 
echocardiographic parameters in defining RV systolic function. Future studies should also determine the ability of RVOT-VTI to predict prognosis and response to therapy, such as tricuspid valve repair or replacement in patients with severe TR and questionable RV function.

\section{REFERENCES}

1. Go AS, Mozaffarian D, Roger VL, Benjamin EJ, Berry JD, Borden WB et al. (2013): Heart disease and stroke statistics - 2013 update: a report from the American Heart Association. Circulation, 127: 6-245.

2. Adams KF, Fonarow GC, Emerman CL, LeJemtel TH, Costanzo RM, Abraham WT et al. (2005): Characteristics and outcomes of patients hospitalized for heartfailure in the United States: rationale, design, and preliminary observationsfrom the first 100,000 cases in the acute Decompensated heart failureNational Registry. Am Heart J., 149: 209-16.

3. Elliott P, Andersson B, Arbustini E et al. (2008): Classification of the cardiomyopathies: a position statement from the EuropeanSociety of Cardiology Working Group on Myocardial and Pericardial Diseases. Eur Heart J., 29: 270-6.

4. Haddad F, Doyle R, Murphy DJ, Hunt SA (2008): Right ventricular function in cardiovascular disease, part II: pathophysiology, clinical importance, and management of right ventricular failure. Circulation, 117: 1717-31.

5. Gorcsan J (2000): Tissue Doppler Echocardiography. Curr Opin Cardiol., 15: 323-329.

6. Amresh R, Zachary M, Paul R (2012): Right Ventricular Outflow Tract Velocity Time Integral is a Strong Predictor of Right Ventricular Dysfunction in Elderly Patients With Severe Aortic Stenosis. Journal of Cardiac Failure, 18 : 8S.

7. Haddad F, Hunt SA, Rosenthal DN et al. (2008): Right ventricular function in cardiovascular disease, part I: Anatomy, physiology, aging, and functional assessment of the right ventricle. Circulation,117:1436-48.

8. Miller LW, Guglin M (2013): Patient selection for ventricular assist devices. J Am Coll Cardiol. ,61:1209-21.

9. Juilliere Y, Barbier G, Feldmann L, Grentzinger A, Danchin N, Cherrier F (1997): Additional predictive value of both left and right ventricular ejection fractions on long-term survival in idiopathic dilated cardiomyopathy. Eur Heart J .,18:276-80.

10.de Groote $P$, Millaire A, Foucher-Hossein $C$ et al. (1998): Right ventricular ejection fraction is an independent predictor of survival in patients with moderate heart failure. J Am Coll Cardiol.,32:948-54.

11.Gavazzi A, Berzuini C, Campana C et al. (1997): Value of right ventricular ejection fraction in predicting short-term prognosis of patients with severe chronic heart failure. J Heart Lung Transplant ., 16:774-85.

12.Gulati A, Ismail TF, Jabbour A et al. (2013): The prevalence and prognostic significance of right ventricular systolic dysfunction in nonischemic dilated cardiomyopathy. Circulation, 128:1623-33.
13.Bursi F, McNallan SM, Redfield MM et al. (2012): Pulmonary pressuresand death in heart failure: a community study.J Am Coll Cardiol .,59:222-31.

14.Damy T, Viallet C, Lairez O, et al. (2009): Comparison of four right ventricular systolic echocardiographic parameters to predict adverse outcomes in chronic heart failure. Eur J Heart Fail.,11:818-24.

15. de Groote P, Fertin M, Goeminne C et al. (2012): Right ventricular systolic function for risk stratification in patients with stable left ventricular systolic dysfunction: comparison of radionuclide angiography to echoDoppler parameters. Eur Heart J.,33:2672-9.

16. La Vecchia L, Zanolla L, Varotto L et al. (2001) : Reduced right ventricular ejection fraction as a marker for idiopathic dilated cardiomyopathy compared with ischemic left ventricular dysfunction. Am Heart J.,142:181-9.

17.McMurray JJ, Adamopoulos S, Anker SD et al. (2012): ESC guidelines for the diagnosis and treatment of acute and chronic heart failure 2012: The Task Force for the Diagnosis and Treatment of Acute and Chronic Heart Failure 2012 of the European Society of Cardiology. Developed in collaboration with the Heart Failure Association (HFA) of the ESC. Eur J Heart Fail .,14:803-69.

18. Lindqvist $P$, Henein $M$, Kazzam E(2003): Right ventricular outflow-tract fractional shortening: an applicable measure of right ventricular systolic function. Eur J Echocardiogr .,4:29-35.

19. Mor-Avi V, Lang RM, Badano LP, Belohlavek M, Cardim NM, Derumeaux G et al. (2011): Current and evolving echocardiographic techniques for the quantitative evaluation of cardiac mechanics: ASE/EAE consensus statement on methodology and indications endorsed by the Japanese Society of Echocardiography. J Am Soc Echocardiogr.,24:277-313.

20. Leather HA, Ama' R, Missant C, Rex S, Rademakers FE, Wouters PF(2006): Longitudinal but not circumferential deformation reflects global contractile function in the right ventricle with open pericardium. Am J Physiol Heart Circ Physiol.,290:H2369-H2375.

21. Puwanant S, Park M, Popović ZB, Tang WH, Farha S, George D et al. (2010): Ventricular geometry, strain, and rotational mechanics in pulmonary hypertension. Circulation, 121:259-266.

22. Utsunomiya H, Nakatani S, Okada T, Kanzaki H, Kyotani S, Nakanishi $N$ et al. (2011): A simple method to predict impaired right ventricular performance and disease severity in chronic pulmonary hypertension using strain rate imaging. Int J Cardiol., 147:88-94.

23. Altekin RE, Karakas MS, Yanikoglu A, Ozel D, Ozbudak O et al. (2012): Determination of right ventricular dysfunction using the speckle tracking echocardiography method in patients with obstructive sleep apnea. Cardiol J., 19:130-139.

24. Meris A, Faletra F, Conca C, Klersy C, Regoli F, Klimusina J et al. (2010): Timing and magnitude of 


$$
\text { ejhm.journals.ekb.eg }
$$

regional right ventricular function: a speckle trackingderived strain study of normal subjects and patients with right ventricular dysfunction. $J$ Am Soc Echocardiogr.,23:823-831.

25. Pedrinelli R, Canale ML, Giannini C, Talini E, Dell'Omo G, Di BelloV (2010) :Abnormal right ventricular mechanics in early systemic hypertension: a two-dimensional strain imaging study. Eur J Echocardiogr.,11:738-742.

26. Teske AJ, De Boeck BW, Olimulder M, Prakken NH, DoevendansPA, Cramer MJ (2008) : Echocardiographic assessment of regional right ventricular function: a headto-head comparison between 2-dimensional and tissue Doppler-derived strain analysis. J Am Soc Echocardiogr.,21:275-283.

27. Rudski LG, Lai WW, Afilalo J, Hua L, Handschumacher MD, Chandrasekaran K et al. (2010) : Guidelines for the echocardiographic assessment of the right heart inadults: a report from the American Society of
Echocardiography endorsed bythe European Association of Echocardiography, a registered branch of the European Society of Cardiology, and the Canadian Society of Echocardiography. J Am Soc Echocardiogr.,23:685-713.

28. Haddad F, Hunt SA, Rosenthal DN, Murphy DJ(2008) : Right ventricular function in cardiovascular disease, part I: Anatomy, physiology, aging, and functional assessmentof the right ventricle. Circulation,117:1436-48.

29. Abraham WT, Adams KF, Fonarow GC, Costanzo MR, Berkowitz RL, LeJemtel TH et al. (2005): Inhospital mortality in patients with acute decompensated heartfailure requiring intravenous vasoactive medications: an analysis from theacute Decompensated heart failure National Registry. J Am Coll Cardiol.,46(1):57.

30. Amin R, Zachary M, Paul R(2012): RVOT VTI as a Strong Predictor of Right Ventricular Dysfunction in Elderly Patients With Severe Aortic stenosis. Journal of Cardiac Failure, 18:85. 